\title{
Research on Key Technologies of Remote Health Intelligent Assisted Diagnosis Based on Power Carrier Communication
}

\author{
Dandan Wang ${ }^{1}$, Fang Liu ${ }^{1}$, Zhuo Huang ${ }^{1}$, Yong Yan ${ }^{2}$ \\ ${ }^{1}$ Unit State Grid Information and Communication Industry Group Co., LTD. Beijing Branch, China \\ ${ }^{2}$ Zhejiang Electric Power Research Institute of State Grid Co., LTD, China
}

Keywords: power carrier communication, channel modeling, electrocardiography, diagnosis.

\begin{abstract}
Power line communication (power line communication, PLC) technology is an important communication method widely used in power systems. The establishment of the power line channel model and the optimization of the power line carrier frequency are of great significance for the realization of high-speed data transmission on the power line. First, the basic theory of power line signal transmission is briefly introduced, and then the classification of the parameters required for modeling is carried out, and various power line channel modeling techniques are summarized. Introduce the development process of various modeling technologies, and analyze the advantages and disadvantages of various technologies. Finally, a power line carrier channel modeling method based on graph theory is proposed, and the feasibility of the method applied to medium voltage power line carrier communication in smart grid is analyzed. Summarizes the development history of intelligent diagnostic technology and the current research status at home and abroad, highlights its development and application in ECG diagnosis, describes various methods and diagnostic processes of ECG intelligent diagnosis, and discusses the development of ECG intelligent diagnosis technology and Outlook.
\end{abstract}

\section{Introduction}

In the past ten years, there have been a large number of practical application research [1] articles such as automatic meter reading system [2] and home automation system based on power carrier communication technology in authoritative journals and international conferences. Power carrier communication is to transmit high-speed data information on the path of the loaded power frequency power signal, so it has the characteristics of harsh working environment, severe interference and large time-varying characteristics fill [3]. Therefore, how to accurately establish an effective power carrier communication channel transmission model, and then determine the best signal transmission frequency according to the frequency selection characteristics [4], is of great theoretical significance and practical value for the development and application support of power carrier communication technology [5].

With the development of technologies such as computers and artificial intelligence, intelligent diagnosis came into being. Intelligent diagnosis technology [6].

This article intends to review the application of intelligent diagnosis in electrocardiography [7].

\section{Power carrier communication model and intelligent diagnosis technology}

\subsection{Principle of high-speed power line carrier communication based on OFDM}

The structure of high-speed power line carrier communication system based on OFDM is shown in Figure 1.

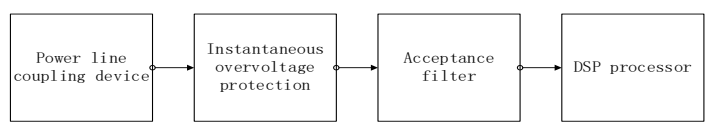

Figure 1 Block diagram of the system 
(1) Sending end processing module

The processing flow at the sending end is shown in Figure 2.

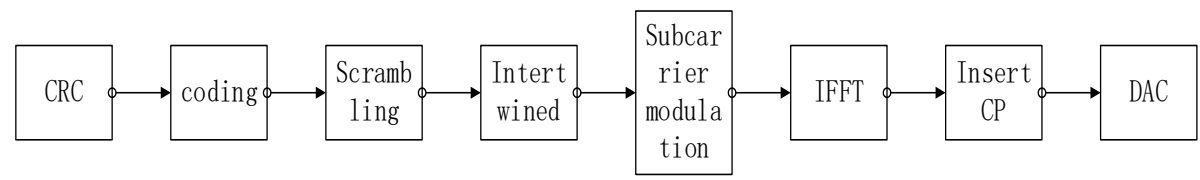

Figure 2 Flow chart of the sending end

1) Polynomial cyclic redundancy coding (CRC).

The stronger the multi-bit error detection capability of the generator polynomial, the more computing and storage resources are required, and vice versa If the calculation complexity is low, you need to use a shorter generator polynomial, and its error detection ability will also decrease[9].

2) Convolutional coding.

The system uses commonly used $(2,1,7)$ convolutional codes to encode the data after CRC processing. The encoder is composed of 2 adders and 6 shift registers, and the input is $\mathrm{x}$ and $\mathrm{y}$. The implementation block diagram is as follows As shown in Figure 3.

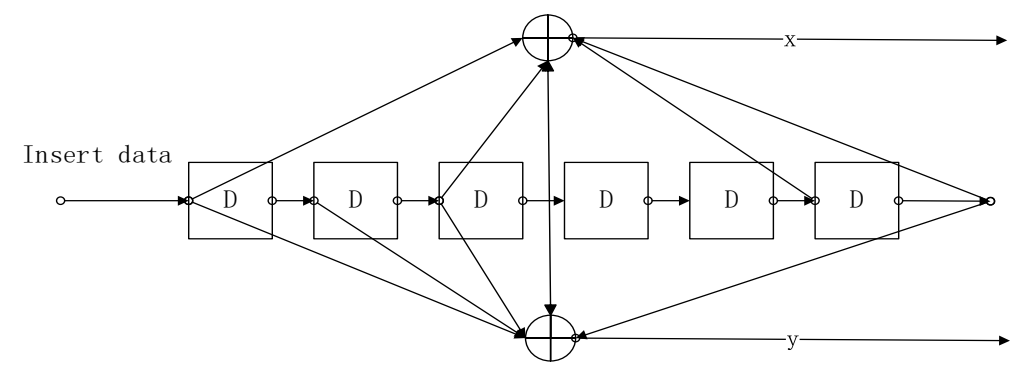

Figure $3(2,1,7)$ Convolutional coding structure diagram

After convolutional coding, the supervising element of each code group not only supervises one information element of the current code group, but also supervises the six information elements of the six code groups.

3) Scrambling.

The scrambling code can randomize the bit stream to reduce the power peak-to-average ratio of the inverse fast Fourier transform (IFFT) output. Scrambling code adopts pseudo-random sequence code, specifically adopts m-sequence PN code, code length is 255 .

\subsection{Receiver processing module}

Figure 4 shows the method of the receiving end processing module.

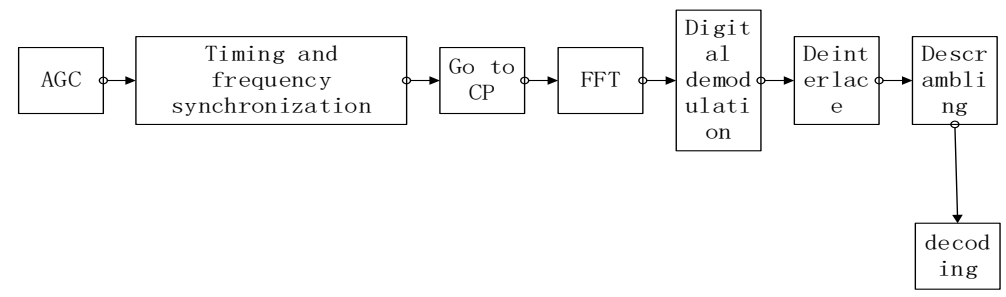

Figure 4 Receiver processing module diagram

Automatic gain control . Due to the large changes in power line impedance and the presence of pulse interference, the received signal has a very wide dynamic range, usually reaching $120 \mathrm{~dB}$. The specific algorithm is as follows: assuming that the received signal is $r$ (n. positive integer) and the length of the sliding window is L, the energy of the received signal in a sliding window can be expressed as

$$
E_{n}=\sum_{k=0}^{L-1} r_{n-k n-k}^{*}=\sum_{k=0}^{L-1}\left|r_{n=k}\right|^{2}
$$


The influence of state noise and interference on the absolute threshold setting adopts the bisector window, and the ratio of the energy of the latter part to the energy of the previous part is used as the decision variable, expressed as

$$
\begin{gathered}
A_{n}=\sum_{k=0}^{L / 2-1} r_{n-k} r_{n-k}^{*}=\sum_{k=0}^{L / 2-1}\left|r_{n-k}\right|^{2} \\
B_{n}=\sum_{k=L / 2}^{L-1} r_{n-k} r_{n-k}^{*}=\sum_{k=L / 2}^{L-1}\left|r_{n-k}\right|^{2} \\
\lambda(n)=B_{n} / A_{n}
\end{gathered}
$$

At the peak point, A. The value of is equal to the noise energy, $B$. The value is the sum of the energy of the signal and noise, so the peak point can be used to calculate the signal-to-noise ratio of the received signal:

$$
\lambda_{\text {peal }}=\frac{B_{\text {peak }}}{A_{\text {peak }}}=\frac{S+N}{N}=S N R+1
$$

The precise synchronization algorithm uses the local chirp signal and the received signal to correlate, and uses the energy of the received signal to normalize the signal, as shown in equation (6):

$$
\gamma(n)=\frac{\left|\sum_{i=0}^{N-1} r[n-i] s_{N-i}^{*}\right|}{\sqrt{\sum_{i=0}^{N-1}|r[n-i]|^{2}}}
$$

In the formula, $\mathrm{s}$ is the discrete chirp signal; $\mathrm{N}$ is the sampling processing flow chart of chirp signal as shown in Figure 5.

\subsection{The principle of intelligent diagnosis technology}

(1) Definition of intelligent diagnosis

Artificial intelligence is a concept proposed by Mc Yi ('arthy et al. in the academic symposium on machine simulation intelligence launched in 1956.

(2) Pattern recognition

When people see an object or phenomenon, by collecting the information of the object or phenomenon, comparing its characteristics with the relevant information already in the mind, finding a same or similar match, then the object or phenomenon can be identified come out. Therefore, an object or phenomenon [12].

A complete pattern recognition system consists of data acquisition, data processing, feature extraction and selection, and classification decision-making components, as shown in Figure 5.

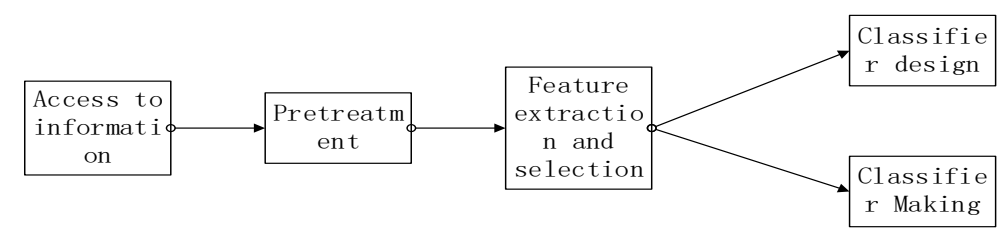

Figure 5 Pattern recognition system

\section{Intelligent diagnosis}

\subsection{The development process of ECG intelligent diagnosis}

In recent years, the number of cardiovascular deaths has increased year by year. In 2012, the number of cardiovascular deaths in my country accounted for \}100:a \} of the total deaths. It has been more than 100 years since Ycc-thovcn used electrocardiography for diagnosis in clinical medicine. 


\section{2 diagnosis method based on expert system}

Through the preliminary analysis of the automatic diagnosis system, in the earliest computer-aided diagnosis stage, the doctor only needs to focus on the electrocardiogram diagnosed as abnormal by the electrocardiograph, thereby reducing the burden on the clinician. But the arrhythmia detected by this method is limited.

\subsection{Diagnostic method based on fuzzy logic}

In the process of disease development, there will be some intermediate states and signs between health and disease, so let the patients' various signs and various diseases should be a vague , the diagnosis requires not only to use "whether there is a disease" To express the results, it is also necessary to give the possibility, location and degree of lesions.Most knowledge is vague knowledge.

\subsection{Diagnostic method based on neural network}

Neural network, as an adaptive pattern recognition technology, does not need to give prior knowledge about the pattern and discriminant function in advance, but automatically forms the required decision area through its own learning mechanism, with parallel distributed processing capabilities, and Self-organization, self-learning, self-adaptive ability and associative memory function. With the emergence and development of neural network analysis algorithms, a variety of neural network technologies have been used to classify electrocardiograms and conduct research on electrocardiogram analysis.

\subsection{Diagnosis method based on neural network and expert system}

Neural network systems and expert systems have their own strengths and weaknesses. In order to achieve the complementarity of advantages, since the 1990s, people have begun to explore those with self-learning capabilities, deep models and deep reasoning mechanisms.

\subsection{Optimization of ECG diagnosis}

The electrocardiogram is the graphic abbreviation of the heart in each cardiac cycle, which is changed by the pacing point. The waveform generated by one beat is called an beat, or heart beat for short.

\subsection{Improve the accuracy of signal acquisition and detection classification}

Preprocessing is to eliminate noise in the input data or information. Human ECG signal is a weak electrical signal with low signal-to-noise ratio. When collecting ECG signals, it will be interfered by various noises. Digital filtering is a common method for enhancing information and suppressing noise.

\subsection{Development of Smart Mobile Healthcare}

Long-term observation and analysis of monitoring data on the heart by mobile electrocardiogram, uploading the heart data through the wireless network, and with the help of big data analysis technology, doctors can perform statistics and calculations on common cases and establish an effective database.

\section{Conclusion}

The application of intelligent diagnosis in electrocardiogram technology has developed rapidly in the past few decades. Some new theories and methods have been greatly developed, and new medical forms have emerged, such as remote intelligent diagnosis based on the development of wireless networks. However, each diagnostic method has its advantages and disadvantages. With the development and maturity of computer technology, artificial intelligence technology, genetic algorithm, artificial neural network technology and other nonlinear technologies, from the perspective of development trends, the model of medical expert system It may be based on a variety 
of intelligent technologies. The currently developed genetic algorithms, fuzzy algorithms, rough set theory and other nonlinear mathematical methods may be combined with artificial neural network technology to construct a new medical expert system model.

\section{Acknowledgements}

Science and Technology Project of State Grid Headquarters: R\&d and application demonstration of key technologies of highly trusted Intelligent perception interactive integrated Service System (No. : 5211DS18002J)

\section{References}

[1] Sherif Y S, Zahir S. On power line carrier communication (PLC)[J]. Microelectronics reliability, 1984, 24(4):781-791.

[2] Whyte I A .Distribution network powerline carrier communication system: US 1976.

[3] Mansfield A R. Method and means of operating a power line carrier communication system [J]. 1991.

[4] Ropp M E , Aaker K , Haigh J , et al. Using power line carrier communications to prevent islanding [of PV power systems][C]// IEEE Photovoltaic Specialists Conference. IEEE, 2000.

[5] M. E. Ropp, K. Aaker, J. Haigh, et al. Using power line carrier communications to prevent islanding [of PV power systems][J]. 2000.

[6] Arthur A. Anderson, Dirk J. Boomgaard, Richard A. Johnson. Power line carrier communication system: US 1989.

[7] XU Wei, WANG Bin, JIANG Yuan-jian. Power Line Carrier Communication Technology and its Applications in Electric Energy Data Acquisition System [J]. Electrical measurement \& instrumentation, 2010.

[8] Kenneth E. Daggett, Dirk J. Boomgaard. Demodulator for powerline carrier communications [D]. US, 1996.

[9] Morgan, D.R, Craig, et al. Adaptive interference cancellation for power line carrier communication systems [J]. IEEE Transactions on Power Delivery, 1991.

[10] Amodeo Guillermo. Power socket incorporating power line carrier communications and physical asset tracking and management system using such socket [J]. 2002.

[11] Fang W, Shi-Bing Z, Zhen-Chao W. Research on Modulation Technology of Low Voltage Power Line Carrier Communication [J]. Video Engineering, 2010:296-298.

[12] Wei S, Cao J, Chen Y, et al. A Canceling Noise Research for Underground Mine Powerline Carrier Communication Based on Adaptive Theory[C]// Power Electronics and Intelligent Transportation System, 2008. PEITS '08. Workshop on. IEEE, 2008. 